\title{
Detection by regular schemes in degree two
}

\author{
Olivier Haution
}

\begin{abstract}
Using Lipman's results on resolution of two-dimensional singularities, we provide a form of resolution of singularities in codimension two for reduced quasi-excellent schemes. We deduce that operations of degree less than two on algebraic cycles are characterised by their values on classes of regular schemes. We provide several applications of this "detection principle", when the base is an arbitrary regular excellent scheme: integrality of the Chern character in codimension less than three, existence of weak forms of the second and third Steenrod squares, Adem relation for the first Steenrod square, commutativity and Poincaré duality for bivariant Chow groups in small degrees. We also provide an application to the possible values of the Witt indices of non-degenerate quadratic forms in characteristic two.
\end{abstract}

\section{Introduction}

Resolution of singularities, which at the moment is only available for $\mathbb{Q}$-schemes, is a fundamental tool for many questions related to algebraic cycles, for example for the study of operations on Chow groups (see for example [Hau12b]). When considering operations of degree at most $n$, it is often sufficient to resolve singularities of codimension at most $n$, that is, given a scheme $X$, to find a proper birational morphism $f: X^{\prime} \rightarrow X$ such that the singular locus of $X^{\prime}$ has codimension greater than $n$. For $n=1$ this remark has been exploited in [Hau13], together with the observation that we can use the normalisation morphism as a resolution of singularities of codimension one. In the present paper, we observe that it is enough to require that $f$ desingularise $X$ up to codimension $n$ (in the terminology of [Tem08, Tem12]), which means that the singular locus of $X^{\prime}$ is mapped to codimension greater than $n$ in $X$. We prove in Theorem 2.1 that desingularisation up to codimension two is possible: any reduced quasi-excellent scheme $X$ admits a proper birational morphism $f: \widetilde{X} \rightarrow X$ mapping the singular locus of $\widetilde{X}$ to a subset of codimension at least three in $X$.

We then provide some applications to algebraic cycles on schemes over a regular base, using Theorem 2.1 as a replacement for resolution of singularities.

It is worth noting that something close to desingularisation up to codimension $n$ was used already by Hironaka in [Hir64] under the name "localization of resolution data"; see [Hir64, Chapter IV, Section 1] (for example Propositions 1 and 2 in loc.cit.). However, both in [Hir64] and [Tem08, Tem12] desingularisation up to codimension $n$ is only used as a subroutine of a

Received 6 August 2014, accepted in final form 15 October 2014.

2010 Mathematics Subject Classification 14C40, 14E15

Keywords: Steenrod operations, Chern character, Resolution of singularities, Bivariant classes, Operational Chow group, Witt indices

This journal is (c) Foundation Compositio Mathematica 2015. This article is distributed with Open Access under the terms of the Creative Commons Attribution Non-Commercial License, which permits non-commercial reuse, distribution, and reproduction in any medium, provided that the original work is properly cited. For commercial re-use, please contact the Foundation Compositio Mathematica. 


\section{DETECTION BY REGULAR SCHEMES IN DEGREE TWO}

global desingularisation method or algorithm; one of the purposes of this paper is to illustrate how this procedure can be used outside of the realm of desingularisation theory.

The paper is organised as follows. In Section 1, we fix the conventions used in the text.

In Section 2, we prove the main result, Theorem 2.1 mentioned above. The strategy for the proof is the following. We may assume that $X$ is normal. Then there are only finitely many singular points $s_{1}, \ldots, s_{n}$ of codimension at most two in $X$. Using resolution of singularities in dimension two, we can resolve each of the local schemes $Y_{i}=\operatorname{Spec} \mathcal{O}_{X, s_{i}}$. The next step is to extend these local resolutions to a proper birational morphism $f: \widetilde{X} \rightarrow X$ inducing an isomorphism outside of the closure of $\left\{s_{1}, \ldots, s_{n}\right\}$. This is possible since a resolution of singularities of $Y_{i}$ may be achieved by the blow-up along a zero-dimensional closed subscheme (we refer to a paper of Lipman for this result $\left.{ }^{1}\right)$. Any singular point $x$ of $\widetilde{X}$ lies over a singular point $f(x)$ of $X$; on the other hand, if $f(x)$ has codimension two in $X$, it is one of the points $s_{1}, \ldots, s_{n}$, and it follows from the construction that $\widetilde{X}$ must be regular at $x$.

In Section 3, we translate the main result into a technical statement (Lemma 3.1) adapted to the situations considered in each of the subsequent sections. It expresses the idea that the vanishing of an operation on algebraic cycles, which lowers the dimension by at most two, is detected by its action on the fundamental classes of regular schemes.

In Section 4, we deduce an integrality property for the Chern character in codimension two, and then show that it actually implies the same property in codimension three. We construct two operations on Chow groups modulo two (and two-torsion for the target), which are related to the second and third Steenrod squares. As an application, we deduce a result contained in Hoffmann's conjecture, concerning Witt indices of quadratic forms; this is a new statement when the base field has characteristic two.

In Section 5, we extend, by descent, the definition of the first Steenrod square $\mathrm{Sq}_{1}$, given in [Hau13b] for quasi-projective varieties, to arbitrary schemes of finite type over a field. We prove the relation $\mathrm{Sq}_{1} \circ \mathrm{Sq}_{1}=0$, which was apparently out of reach of the techniques used in [Hau13b]. The argument, as well as the construction of $\mathrm{Sq}_{1}$, does not depend on the characteristic of the base field; in characteristic different from two, a different construction of the operation $\mathrm{Sq}_{1}$ has been given in [Bro03, Voe03], and the relation $\mathrm{Sq}_{1} \circ \mathrm{Sq}_{1}=0$ was known, being one of the Adem relations.

In Section 6, we discuss consequences for the bivariant Chow group. We prove an absolute form of Poincaré duality, which implies that the operational and usual Chow groups of a regular excellent scheme coincide in degrees at most two. We also discuss commutativity of bivariant classes of small degree; in particular, we prove that any class of degree at most two commuting with proper push-forwards, and pull-backs along smooth morphisms and regular closed embeddings automatically commutes with flat pull-backs. Here resolution of singularities is combined with another, a priori independent, feature of codimension at most two: bivariant classes of degree at most two can be expressed using Chern classes with supports. In higher degrees, denominators appear, and one needs other sources of bivariant classes, such as $K$-cohomology. Finally, we explain how these results can be extended to classes of arbitrary degrees in characteristic zero, where both resolution of singularities and Gersten's conjecture are available.

\footnotetext{
${ }^{1}$ As pointed out by the referee, the minimal desingularisation input that we need is the following: if $X$ is a quasiexcellent two-dimensional normal local scheme, then there exist a regular scheme $X^{\prime}$ and a proper birational morphism $X^{\prime} \rightarrow X$ which induces an isomorphism over the complement of the closed point in $X$.
} 


\section{Olivier Haution}

\section{Terminology and conventions}

\subsection{Schemes and morphisms}

All schemes are noetherian and separated. When $S$ is a scheme, we denote by $\mathrm{Sch}_{S}$ the category of schemes of finite type over $S$ and proper $S$-morphisms.

We say that a morphism $Y \rightarrow X$ is, or induces, an isomorphism outside of a closed subset $Z$ of $X$ if the base change $(X-Z) \times_{X} Y \rightarrow X-Z$ is an isomorphism.

\subsection{Regularity}

We say that a scheme $X$ is regular at a point $x$ when the local ring $\mathcal{O}_{X, x}$ is regular. Otherwise we say that $x$ is a singular point of $X$. The singular locus $\operatorname{Sing} X$ is the set of such points. A scheme is regular if it is regular at each of its points.

\subsection{Normalisation}

When $X$ is an integral scheme, its normalisation $X^{\prime} \rightarrow X$ is the affine morphism given locally by the integral closure of its coordinate ring in its function field. More generally, when $X$ is an arbitrary scheme, its normalisation is the composition $\coprod_{i} X_{i}^{\prime} \rightarrow \coprod_{i} X_{i} \rightarrow X$, where the $X_{i}$ are the irreducible components of $X$, and $X_{i}^{\prime} \rightarrow X_{i}$ their normalisations.

\subsection{Excellence}

A scheme $S$ is called quasi-excellent if

- any integral scheme finite over $S$ admits an open dense regular subscheme,

- and for any closed point $x$ of $X$, the completion morphism of $\operatorname{Spec} \mathcal{O}_{X, x}$ has geometrically regular fibres.

If $S$ is quasi-excellent, then any scheme of finite type over $S$ is quasi-excellent, and so is the localisation of $S$ at any of its points [ILO12, $\S 2$, Theorem 5.1]. The spectrum of a field, and of $\mathbb{Z}$, is quasi-excellent, so that the class of quasi-excellent schemes contains all varieties and arithmetic schemes. The normalisation of a quasi-excellent scheme is finite [EGA IV, (7.6.1)]; the singular locus of a quasi-excellent scheme is closed [EGA IV, (6.12.3)]. For a scheme of finite type over a regular scheme, we will use the terminology excellent instead of quasi-excellent; this is compatible with [EGA IV, (7.8.2)].

\subsection{Projectivity}

A morphism $Y \rightarrow X$ is projective if there are a quasi-coherent sheaf of graded $\mathcal{O}_{X}$-algebras $\mathcal{S}_{\bullet}$, with $\mathcal{S}_{1}$ a coherent $\mathcal{O}_{X}$-module generating $\mathcal{S}_{\boldsymbol{\bullet}}$ as an $\mathcal{O}_{X}$-algebra, and an isomorphism over $X$ between $Y$ and $\operatorname{Proj}_{X} \mathcal{S}_{\bullet}$. A morphism is quasi-projective if it decomposes as an open immersion followed by a projective morphism.

\subsection{Envelopes}

A proper morphism $f: Y \rightarrow X$ is an envelope if for any integral closed subscheme $Z$ of $X$, there is a closed subscheme $W$ of $Y$ such that $f$ induces a birational morphism $W \rightarrow Z$. It is equivalent to require that $Y(K) \rightarrow X(K)$ be surjective for all fields $K$. When $X$ is of finite type over $S$, a Chow envelope over $S$ is an envelope $Y \rightarrow X$ with $Y$ quasi-projective over $S$. By Chow's Lemma [EGA II, (5.6.1)] and noetherian induction, such an envelope always exists. 


\section{DETECTION BY REGULAR SCHEMES IN DEGREE TWO}

\subsection{Dimension}

The codimension $\operatorname{codim}_{X}(x)$ of a point $x$ in a scheme $X$ is the dimension of the local ring $\mathcal{O}_{X, x}$. When $Y$ is a closed subset of $X$, its codimension $\operatorname{codim}_{X}(Y)$ is the infimum of the codimensions in $X$ of the points of $Y$. When $X$ is an integral scheme of finite type over a scheme $S$, the dimension of $X$ over $S$ is defined as

$$
\operatorname{dim}_{S} X=\operatorname{tr} . \operatorname{deg} .(\kappa(X) / \kappa(\bar{X}))-\operatorname{codim}_{S}(\bar{X}),
$$

where $\bar{X}$ is the scheme-theoretic image of $X \rightarrow S$, and $\kappa(X)$ and $\kappa(\bar{X})$ are the function fields of $X$ and $\bar{X}$, respectively. When $Z$ is an integral closed subscheme of $X$, we have, using the dimension formula [EGA IV, (5.6.5.1)],

$$
\begin{aligned}
\operatorname{dim}_{S} Z & =\operatorname{tr} \cdot \operatorname{deg} \cdot(\kappa(Z) / \kappa(\bar{Z}))-\operatorname{codim}_{S}(\bar{Z}) \\
& \leqslant \text { tr.deg. }(\kappa(Z) / \kappa(\bar{Z}))-\operatorname{codim}_{\bar{X}}(\bar{Z})-\operatorname{codim}_{S}(\bar{X}) \\
& \leqslant \text { tr.deg. }(\kappa(X) / \kappa(\bar{X}))-\operatorname{codim}_{X}(Z)-\operatorname{codim}_{S}(\bar{X}) \\
& =\operatorname{dim}_{S} X-\operatorname{codim}_{X}(Z) .
\end{aligned}
$$

In particular, $\operatorname{dim}_{S} Z \leqslant \operatorname{dim}_{S} X$, so that when $Y$ is a scheme of finite type over $S$, we may define $\operatorname{dim}_{S} Y$ as the supremum of the integers $\operatorname{dim}_{S} Z$ for $Z$ an integral closed subscheme of $Y$.

Let $X$ be an integral scheme of finite type over $S$ and let $Y$ be a closed subscheme of $X$. Since for any integral closed subscheme $Z$ of $Y$, we have $\operatorname{dim}_{S} Z \leqslant \operatorname{dim}_{S} X-\operatorname{codim}_{X}(Z) \leqslant$ $\operatorname{dim}_{S} X-\operatorname{codim}_{X}(Y)$, it follows that

$$
\operatorname{dim}_{S} Y \leqslant \operatorname{dim}_{S} X-\operatorname{codim}_{X}(Y) .
$$

\subsection{Abelian groups}

We denote by $\mathrm{Ab}$ the category of abelian groups. Let $p$ be a prime number. We denote by $\mathbb{Z}_{(p)}$ the subgroup of $\mathbb{Q}$ consisting of those fractions whose denominator is prime to $p$. When $A$ is an abelian group and $a \in A \otimes_{\mathbb{Z}} \mathbb{Q}$, we let $v_{p}(a)$ be the largest integer $k$ such that $p^{-k} \cdot a$ belongs to the image of $A \otimes_{\mathbb{Z}} \mathbb{Z}_{(p)} \rightarrow A \otimes_{\mathbb{Z}} \mathbb{Q}$ (we set $v_{p}(0)=\infty$ ). When $t$ is an integer, the group $A \otimes_{\mathbb{Z}}\left(\mathbb{Q} /\left(p^{-t} \cdot \mathbb{Z}_{(p)}\right)\right)$ may be identified with the quotient of the group $A \otimes_{\mathbb{Z}} \mathbb{Q}$ by the subgroup of elements $a$ satisfying $v_{p}(a) \geqslant-t$.

\subsection{Chow groups, Grothendieck groups}

Let $S$ be a regular scheme. We denote by $K_{0}^{\prime}: \mathrm{Sch}_{S} \rightarrow$ Ab the functor given by the Grothendieck group of coherent sheaves. For an object $X$ of $\operatorname{Sch}_{S}$, the subgroup $K_{0}^{\prime}(X)_{(d)} \subset K_{0}^{\prime}(X)$ is generated by the elements $\left[\mathcal{O}_{Z}\right]$, where $Z$ is a closed subscheme of $X$ with $\operatorname{dim}_{S} Z \leqslant d$ (see 1.7). The Chow group of cycles of dimension $j$ over $S$ is a functor $\mathrm{CH}_{j}: \mathrm{Sch}_{S} \rightarrow \mathrm{Ab}$; see [Ful98, $\left.\S 20\right]$. We have the $j$ th homological Chern character, a natural transformation (this is the $j$ th component of the morphism $\tau$ of [Ful98, Theorem 18.3, § 20])

$$
\operatorname{ch}_{j}: K_{0}^{\prime}(-) \rightarrow \mathrm{CH}_{j}(-) \otimes_{\mathbb{Z}} \mathbb{Q} .
$$

\section{Resolving singularities in codimension two}

TheOrem 2.1. Let $X$ be a reduced quasi-excellent scheme. Then there are a reduced scheme $\widetilde{X}$ and a projective birational morphism $f: \widetilde{X} \rightarrow X$ with the following properties.

(i) The scheme $\tilde{X}$ is regular at any of its points $x$ such that $f(x)$ has codimension at most 


\section{OLIVIER HAUtion}

two in $X$.

(ii) The morphism $f$ is an isomorphism outside of the singular locus of $X$.

Proof. We first assume that $X$ is normal. Then $\operatorname{Sing} X$ contains no point of codimension at most one in $X$. Let $\Sigma$ be the set of points of $\operatorname{Sing} X$ which have codimension two in $X$. We view $\Sigma$ and $\operatorname{Sing} X$ as topological subspaces of $X$. Then $\Sigma$ consists of generic points of $\operatorname{Sing} X$. Since $X$ is quasi-excellent, $\operatorname{Sing} X$ is the support of a noetherian scheme, and therefore the space $\Sigma$ is finite and discrete. Thus we can write $\Sigma=\left\{s_{1}, \cdots, s_{n}\right\}$, with $s_{i}$ not belonging to the closure of $\left\{s_{j} \mid j \neq i\right\}$ in $X$.

For every $i \in\{1, \ldots, n\}$, the scheme $Y_{i}=\operatorname{Spec} \mathcal{O}_{X, s_{i}}$ is a singular normal quasi-excellent scheme of dimension two. By [Lip78, Theorem p. 151, and C p. 155], there is a zero-dimensional closed subscheme $Z_{i}$ of $Y_{i}$ such that the blow-up $g_{i}: \widetilde{Y}_{i} \rightarrow Y_{i}$ of $Y_{i}$ along $Z_{i}$ is a desingularisation; that is, $\widetilde{Y}_{i}$ is regular.

We let $Z=\coprod_{i=1}^{n} Z_{i}$ and $Y=\coprod_{i=1}^{n} Y_{i}$, and claim that $z: Z \rightarrow Z \times_{X} Y$ is an isomorphism. Indeed, since the open subschemes $Z_{j} \times_{X} Y_{i}$ for $i, j=1, \ldots, n$ cover $Z \times_{X} Y$, it suffices to see that the base change $z_{i, j}: Z_{j} \times_{Y} Y_{i} \rightarrow Z_{j} \times_{X} Y_{i}$ of $z$ is an isomorphism. If $j \neq i$, no point of $Y_{i}$ is mapped to $s_{j}$, so that $Z_{i} \times_{X} Y_{j}=\emptyset$ and $z_{i, j}$ is an isomorphism. The morphism $Y_{i} \rightarrow X$ is a monomorphism by [EGA I, I (2.4.2)], and so is $Y_{i} \rightarrow Y$. Therefore $d_{i}: Z_{i} \rightarrow Z_{i} \times_{Y} Y_{i}$ and $z_{i, i} \circ d_{i}: Z_{i} \rightarrow Z_{i} \times_{X} Y_{i}$ are isomorphisms, and we deduce that $z_{i, i}$ is an isomorphism.

Let $W$ be the scheme-theoretic image of $Z \rightarrow X$ and let $f: \widetilde{X} \rightarrow X$ be the blow-up of $X$ along $W$. The morphism $Y \rightarrow X$ is flat, and by the compatibility of scheme-theoretic images with flat base change [EGA IV, (2.3.2)], the scheme-theoretic image of the morphism $Z=Y \times_{X} Z \rightarrow Y$ is $Y \times_{X} W$. Since this morphism is a closed embedding, it follows that $Z \rightarrow Y \times_{X} W$ is an isomorphism. By the compatibility of blow-ups with flat base change, we see that the base change of $f$ along $Y \rightarrow X$ is the blow-up of $Y$ along $Z$. In particular, we have a cartesian square, for every $i \in\{1, \ldots, n\}$,

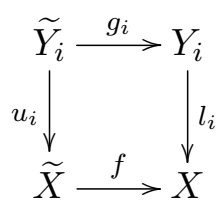

The set-theoretic image of $Z \rightarrow X$ is $\Sigma$ (this is where we use that each $Z_{i}$ is zero-dimensional), hence $W$ is supported on the closure of $\Sigma$, which is contained in $\operatorname{Sing} X$. Therefore $f$ is an isomorphism outside of $\operatorname{Sing} X$, and in particular is birational. It is projective, as is any blow-up. Moreover, $\widetilde{X}$ is reduced, being the blow-up of a reduced scheme.

Thus, to conclude the proof in the normal case, it will suffice to verify condition (i). So let $x$ be a point of $\widetilde{X}$ such that $f(x)$ has codimension at most two in $X$. If $f(x) \notin \Sigma$, then $f(x) \notin \operatorname{Sing} X$ by construction of $\Sigma$. Therefore $f$ induces an isomorphism $\mathcal{O}_{X, f(x)} \rightarrow \mathcal{O}_{\widetilde{X}, x}$, and it follows that $\widetilde{X}$ is regular at $x$. Now assume that $f(x)=s_{i}$ for some $i \in\{1, \ldots, n\}$. Let $F$ be the residue field of $\widetilde{X}$ at $x$. Composing the $F$-point given by $x$ with the morphism $f$, we obtain an $F$-point of $X$. Since its image is the point $s_{i}$, this $F$-point factors through the localisation $l_{i}: Y_{i} \rightarrow X$. In view of the cartesian square (2), we obtain an $F$-point $y$ of $\widetilde{Y}_{i}$ such that $x=u_{i}(y)$. Since $\widetilde{Y}_{i}$ is regular at $y$, and $u_{i}$ is flat, it follows from [EGA IV, $(6.5 .2,(\mathrm{i}))$ ] that $\widetilde{X}$ is regular at $x$.

Now let $X$ be an arbitrary reduced quasi-excellent scheme. Its normalisation $\pi: X^{\prime} \rightarrow X$ is finite and birational. Applying the construction above to the quasi-excellent normal scheme $X^{\prime}$, we obtain a projective birational morphism $f^{\prime}: \widetilde{X} \rightarrow X^{\prime}$ satisfying conditions (i) and (ii). The 


\section{DETECTION BY REGULAR SCHEMES IN DEGREE TWO}

morphism $f=\pi \circ f^{\prime}$ is projective and birational. Since $\pi$ induces an isomorphism outside of $\operatorname{Sing} X$, and $f^{\prime}$ induces an isomorphism outside of $\operatorname{Sing} X^{\prime} \subset \pi^{-1} \operatorname{Sing} X$, we see that $f$ satisfies condition (ii). To see that $f$ satisfies condition (i), let $x$ be a point of $\widetilde{X}$ such that $f(x)$ has codimension at most two in $X$. By Lemma 2.4 below applied to the morphism $\pi$, the point $f^{\prime}(x)$ has codimension at most two in $X^{\prime}$. Since $f^{\prime}$ satisfies (i), it follows that $\widetilde{X}$ is regular at $x$.

Remark 2.2. From the proof, we see that we may take for $f$ the normalisation of $X$, followed by the blow-up along a closed subscheme of codimension two.

We will be using the following variant of Theorem 2.1.

Corollary 2.3. Let $X$ be a reduced quasi-excellent scheme, of finite type over a scheme $S$. Then there is a projective birational morphism $f: X^{\prime} \rightarrow X$, with $X^{\prime}$ reduced and quasi-projective over $S$, and regular at any of his points $x$ such that $f(x)$ has codimension at most two in $X$.

Proof. Applying Chow's lemma [EGA II, (5.6.1)], we obtain a projective birational morphism $Y \rightarrow X$ with $Y$ reduced and quasi-projective over $S$. By Lemma 2.4, a point has codimension at most two in $Y$ as soon as its image has codimension at most two in $X$. The statement follows by applying Theorem 2.1 to the scheme $Y$.

Lemma 2.4. Let $f: Y \rightarrow X$ be a birational morphism of finite type, and let $y$ be a point of $Y$ with image $x=f(y)$ in $X$. Then we have $\operatorname{codim}_{Y}(y) \leqslant \operatorname{codim}_{X}(x)$.

Proof. Let $Y_{0}$ be an irreducible component of $Y$ containing $y$ and such that $\operatorname{codim}_{Y}(y)=$ $\operatorname{codim}_{Y_{0}}(y)$. There is a unique irreducible component $X_{0}$ of $X$ such that $f$ induces a birational morphism $f_{0}: Y_{0} \rightarrow X_{0}$ of finite type. Applying the dimension formula [EGA IV, (5.6.5.1)] to the morphism $f_{0}$, we obtain $\operatorname{codim}_{Y_{0}}(y) \leqslant \operatorname{codim}_{X_{0}}(x)$, and we conclude since $\operatorname{codim}_{X_{0}}(x) \leqslant$ $\operatorname{codim}_{X}(x)$.

\section{Detection by regular schemes}

Let $T \rightarrow S$ be a morphism of finite type and let $F$ be a functor $\operatorname{Sch}_{T} \rightarrow$ Ab. When $f$ is a morphism in $\mathrm{Sch}_{T}$, we will write $f_{*}$ for $F(f)$. We consider the following conditions on $F$, where all schemes are of finite type over $T$ and all morphisms are over $T$.

(F1) For every open immersion $u: U \rightarrow X$, a morphism of abelian groups $u^{*}: F(X) \rightarrow F(U)$ is given.

(F2) If $i$ is a closed embedding, and $u$ the open immersion of its complement, we have an inclusion $\operatorname{ker} u^{*} \subset \operatorname{im} i_{*}$.

(F3) For any cartesian square

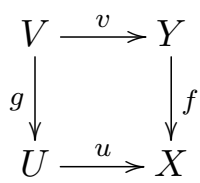

with $u$ an open immersion and $f$ a proper morphism, we have $u^{*} \circ f_{*}=g_{*} \circ v^{*}$.

(F4) If $X$ is integral or empty, we have a specified element $1_{X} \in F(X)$ such that:

(F4a) If $u: U \rightarrow X$ is an open immersion, then $u^{*}\left(1_{X}\right)=1_{U}$.

(F4b) If $f: Y \rightarrow X$ is an isomorphism, then $f_{*}\left(1_{Y}\right)=1_{X}$. 


\section{OLIVIER HAUtion}

(F5) The group $F(X)$ is generated by the elements $f_{*}\left(1_{Y}\right)$ where $Y$ is integral, the morphism $f: Y \rightarrow X$ is proper, and $\operatorname{dim}_{S} Y \leqslant \operatorname{dim}_{S} X$ (see 1.7).

Lemma 3.1. Let $T$ be a scheme of finite type over a quasi-excellent scheme $S$. Let $G, H$ be two functors $\mathrm{Sch}_{T} \rightarrow \mathrm{Ab}$ satisfying (F1) and (F2). Assume that $G$ additionally satisfies (F3), (F4), and (F5). Let $d$ be an integer such that, for every scheme $X$ of finite type over $T$, the following conditions are satisfied.

(i) The group $G(X)$ is generated by the elements $f_{*}\left(1_{Y}\right)$, where $Y$ is integral, the morphism $f: Y \rightarrow X$ is proper, and $\operatorname{dim}_{S} Y \leqslant d$.

(ii) When $\operatorname{dim}_{S} X<d-2$, we have $H(X)=0$.

Assume that, for every scheme $X$ of finite type over $T$, we have a morphism $\rho_{X}: G(X) \rightarrow$ $H(X)$ satisfying the following conditions.

(iii) If $f: Y \rightarrow X$ is a proper morphism, then $f_{*} \circ \rho_{Y}=\rho_{X} \circ f_{*}$.

(iv) If $u: U \rightarrow X$ is an open immersion, then $u^{*} \circ \rho_{X}=\rho_{U} \circ u^{*}$.

(v) For any regular $T$-scheme $R$, quasi-projective over $S$, we have $\rho_{R}\left(1_{R}\right)=0$.

Then $\rho_{X}=0$ for every scheme $X$ of finite type over $T$.

Proof. Assuming the contrary, there is by (i) and (iii) an integral scheme $X$ of finite type over $T$ with $\operatorname{dim}_{S} X \leqslant d$ and $\rho_{X}\left(1_{X}\right) \neq 0$. Using Corollary 2.3, we find a proper birational morphism $f: X^{\prime} \rightarrow X$, with $X^{\prime}$ integral and quasi-projective over $S$, and such that $f$ maps singular points of $X^{\prime}$ to codimension at least three in $X$. By the quasi-excellence of $X^{\prime}$, its singular locus is closed; we let $i$ be the embedding of the associated reduced closed subscheme of $X^{\prime}$, and let $r: R \rightarrow X^{\prime}$ be its open complement. Using successively (iv), (F4a), and (v), we have

$$
r^{*} \circ \rho_{X^{\prime}}\left(1_{X^{\prime}}\right)=\rho_{R} \circ r^{*}\left(1_{X^{\prime}}\right)=\rho_{R}\left(1_{R}\right)=0 .
$$

By (F2) with $F=H$, this implies that $\rho_{X^{\prime}}\left(1_{X^{\prime}}\right) \in \operatorname{im} i_{*}$. Let $z: Z \hookrightarrow X$ be the closed embedding of the scheme-theoretic image of $f \circ i$. Since $Z$ has codimension at least three in $X$, we have $\operatorname{dim}_{S} Z \leqslant \operatorname{dim}_{S} X-3<d-2$ by (1). Therefore $H(Z)=0$ by (ii); hence using (iii), we have

$$
\rho_{X} \circ f_{*}\left(1_{X^{\prime}}\right)=f_{*} \circ \rho_{X^{\prime}}\left(1_{X^{\prime}}\right) \in \operatorname{im}(f \circ i)_{*} \subset \operatorname{im} z_{*}=0 .
$$

Now let $U$ be a non-empty open subscheme of $X$ over which $f$ is an isomorphism, and form the square of (F3). By (F3), (F4a), and (F4b), we have

$$
u^{*}\left(f_{*}\left(1_{X^{\prime}}\right)-1_{X}\right)=u^{*} \circ f_{*}\left(1_{X^{\prime}}\right)-u^{*}\left(1_{X}\right)=g_{*} \circ v^{*}\left(1_{X^{\prime}}\right)-1_{U}=g_{*}\left(1_{V}\right)-1_{U}=0 .
$$

Hence by (F2) with $F=G$, we have $f_{*}\left(1_{X^{\prime}}\right)-1_{X} \in \operatorname{im} j_{*}$, where $j: Q \hookrightarrow X$ is the closed embedding of a complement of $u$. Since $\rho_{X}\left(1_{X}\right) \neq 0$ and $\rho_{X} \circ f_{*}\left(1_{X^{\prime}}\right)=0$, we have $\rho_{X} \circ j_{*} \neq 0$. It follows from (F5) and (iii) that we may find a proper morphism $Y \rightarrow Q$ with $Y$ integral and $\operatorname{dim}_{S} Y \leqslant \operatorname{dim}_{S} Q<\operatorname{dim}_{S} X$ (the last inequality follows from (1)), such that $\rho_{Y}\left(1_{Y}\right) \neq 0$.

Thus we construct by induction (letting $X_{-1}=X$ ) for every $n \in \mathbb{N}$ a proper morphism $X_{n} \rightarrow X_{n-1}$, with $X_{n}$ integral, $\operatorname{dim}_{S} X_{n}<\operatorname{dim}_{S} X_{n-1}$, and $\rho_{X_{n}}\left(1_{X_{n}}\right) \neq 0$. The images of $X_{n}$ in $S$ form a decreasing sequence of closed subsets whose codimensions tend to infinity. This is impossible since $S$ is noetherian.

REMARK 3.2. Assume that $S$ is a $\mathbb{Q}$-scheme. Using resolution of singularities for $\mathbb{Q}$-schemes [Tem08] instead of Theorem 2.1, we can remove conditions (ii) and (iv) in the statement of Lemma 3.1. 


\section{DETECTION BY REGULAR SCHEMES IN DEGREE TWO}

We now describe how the Chow group and the Grothendieck group (see 1.9) give rise to the functors $G$ and $H$ of Lemma 3.1 when $S$ is regular.

ExAmple 3.3. Let $C$ be an abelian group and let $j$ be an integer. The functor $G=H=$ $\mathrm{CH}_{j}(-) \otimes_{\mathbb{Z}} C$ satisfies conditions (F1) to (F5) (with $1_{X}=[X]$ when $\operatorname{dim}_{S} X=j$ and $1_{X}=0$ otherwise). Moreover, conditions (i) and (ii) of Lemma 3.1 are satisfied when $j \leqslant d$ and $j \geqslant d-2$, respectively.

EXAmple 3.4. We say that a morphism $P \rightarrow T$ of finite type has relative dimension at most $r$ if for every scheme $Y$ of finite type over $T$, we have $\operatorname{dim}_{S}\left(P \times_{T} Y\right)-\operatorname{dim}_{S} Y \leqslant r$. Examples of such morphisms are given by closed embeddings $(r=0)$ and flat morphisms of constant relative dimension $r$.

Let $j$ be an integer, and $P \rightarrow T$ a morphism of relative dimension at most $r$. For a scheme $X$ of finite type over $T$, we let $H(X)=\mathrm{CH}_{j+r}\left(X \times_{T} P\right)$. This gives a functor $H: \mathrm{Sch}_{T} \rightarrow \mathrm{Ab}$ satisfying (F1), (F2), and condition (ii) of Lemma 3.1 when $j \geqslant d-2$.

ExAmple 3.5. The functor $G=K_{0}^{\prime}(-)_{(d)}$ (see 1.9) satisfies conditions (F1) to (F5) if we let $1_{X}=\left[\mathcal{O}_{X}\right]$ when $\operatorname{dim}_{S} X \leqslant d$ and $1_{X}=0$ otherwise. In addition, condition (i) of Lemma 3.1 is satisfied.

\section{Integrality of the Chern character}

We formulated in [Hau12a] the following conjecture, which depends on a prime number $p$ and an integer $n$ (see 1.8 and 1.9 for the relevant definitions).

Conjecture 4.1. Let $S$ be a regular scheme, $X$ a scheme of finite type over $S, d$ an integer, and $x \in K_{0}^{\prime}(X)_{(d)}$. We have, for all $i \leqslant n$,

$$
v_{p}\left(\operatorname{ch}_{d-i} x\right) \geqslant-\left[\frac{i}{p-1}\right]
$$

We proved Conjecture 4.1 when $n \leqslant p(p-1)-1$ and $S$ is of finite dimension in [Hau12a, Proposition 3.1 and Appendix].

Theorem 4.2. Conjecture 4.1 is true for $p=2$ and $n=2$ when $S$ is excellent.

Proof. We take $G$ as in Example 3.5, and $H$ as in Example 3.3 (with $j=d-i$ and $C=$ $\mathbb{Q} /\left(2^{-2} \cdot \mathbb{Z}_{(2)}\right)$, see 1.8$)$, and check the conditions of Lemma 3.1 for $T=S$ and the operation

$$
\rho_{X}=\operatorname{ch}_{d-i}: G(X)=K_{0}^{\prime}(X)_{(d)} \rightarrow H(X)=\mathrm{CH}_{d-i}(X) \otimes_{\mathbb{Z}} \mathbb{Q} /\left\{y \mid v_{2}(y) \geqslant-2\right\} .
$$

Conditions (iii) and (iv) follow from [Ful98, Theorem 18.3 (1), (4), and $\S 20]$, while condition (v) is Lemma 4.3 below.

Lemma 4.3. Let $X$ and $S$ be regular schemes, with $X$ quasi-projective over $S$. Then for any integer $i$ and any prime number $p$, we have

$$
v_{p}\left(\operatorname{ch}_{\operatorname{dim}_{S} X-i}\left[\mathcal{O}_{X}\right]\right) \geqslant-\left[\frac{i}{p-1}\right] \text {. }
$$

Proof. We apply Lemma 4.4. The lemma can then be proved using the Riemann-Roch theorem of [Ful98, § 20]; see the proof of [Hau12a, Proposition 4.1]. 


\section{OLIVIER HAUtion}

Lemma 4.4. Let $S$ be a regular scheme. Any quasi-projective morphism $X \rightarrow S$ decomposes as $p \circ i$, with $i$ a closed embedding and $p$ a smooth quasi-projective morphism. If $X$ is regular, then $i$ is a regular closed embedding.

Proof. By [SGA 6, II, Corollary 2.2.7.1], the scheme S, being separated and regular, is divisorial (that is, possesses an ample family). It follows from [SGA 6, Proposition 2.2.3, Lemma 2.1.1 a)] that any coherent sheaf of $\mathcal{O}_{S}$-modules is a quotient of a locally free coherent sheaf. Thus any projective morphism with target $S$ factors as a closed immersion into a projective bundle over $S$. In particular, $X \rightarrow S$ decomposes as $X \stackrel{u}{\rightarrow} Z \stackrel{j}{\rightarrow} P \stackrel{q}{\rightarrow} S$, with $u$ an open immersion, $j$ a closed embedding, and $q$ a smooth projective morphism. Let $T$ be the set-theoretic closed complement of $X$ in $Z$, and let $W$ be the open complement of $j(T)$ in $P$. Then $j$ restricts to a closed embedding $i: X \hookrightarrow W$, and the composition $p: W \rightarrow P \rightarrow S$ is smooth and quasi-projective.

The last statement follows from [EGA IV, (19.1.1)] since $W$ is regular, being smooth over a regular scheme [EGA IV, (17.5.8, (iii))].

Theorem 4.5. Conjecture 4.1 is true for $p=2$ and $n=3$ when $S$ is excellent and of finite dimension.

Proof. Let $X^{\prime} \rightarrow X$ be a Chow envelope over $S$ (see 1.6). Since the morphism $K_{0}^{\prime}\left(X^{\prime}\right)_{(d)} \rightarrow$ $K_{0}^{\prime}(X)_{(d)}$ is surjective, we may replace $X$ with $X^{\prime}$, and assume that $X$ is quasi-projective over $S$. We may also easily reduce to the case when $S$ is connected. Let $x \in K_{0}^{\prime}(X)_{(d)}$, and write $\alpha=\psi_{-1}(x)-(-1)^{-d} \cdot x$. Here $\psi_{-1}$ is the $(-1)$ st homological Adams operation (corresponding to duality theory); see [Sou85, Theorem 7] where $S$ is assumed to be of finite dimension. We have in $\mathrm{CH}_{d-3}(X) \otimes_{\mathbb{Z}} \mathbb{Q}$, by [Hau12a, Proposition 2.4 and Appendix],

$$
\begin{aligned}
\operatorname{ch}_{d-3} \alpha & =\operatorname{ch}_{d-3}\left(\psi-1(x)-(-1)^{d} \cdot x\right) \\
& =(-1)^{3-d} \cdot \operatorname{ch}_{d-3} x-(-1)^{-d} \cdot \operatorname{ch}_{d-3} x \\
& =2 \cdot(-1)^{1-d} \cdot \operatorname{ch}_{d-3} x .
\end{aligned}
$$

Since $\alpha \in K_{0}^{\prime}(X)_{(d-1)}$ by [Hau12a, Proposition A.1], we have $v_{2}\left(\operatorname{ch}_{d-3} \alpha\right) \geqslant-2$ by Theorem 4.2. It follows that $v_{2}\left(\operatorname{ch}_{d-3} x\right) \geqslant-3$, as required.

REMARK 4.6. The arguments used in the proof of Theorem 4.5 are those of the proof of [Hau12a, Theorem 3.2]. More generally (starting the induction $d=n$ instead of $d=0$ in the proof of [Hau12a, Theorem 3.2]), we have:

Fix a prime number $p$. Assume that Conjecture 4.1 is true for $n=m$, with $S$ of finite dimension. Let $m^{\prime}$ be the smallest multiple of $p(p-1)$ such that $m^{\prime}>m$. Then Conjecture 4.1 is true for $n=m^{\prime}-1$.

REMARK 4.7. Using Remark 3.2 and proceeding as in the proof of Theorem 4.2, we see that Conjecture 4.1 is true for any $p$ and $n$ when $S$ is an excellent $\mathbb{Q}$-scheme.

Let $S$ be a regular excellent scheme. By [Hau12a, Section 5], Theorem 4.2, and Theorem 4.5, we obtain morphisms of functors $\mathrm{Sch}_{S} \rightarrow \mathrm{Ab}$

$$
T_{2}: \mathrm{CH}_{\bullet}(-) \otimes_{\mathbb{Z}} \mathbb{Z} / 2 \rightarrow\left(\mathrm{im}\left(\mathrm{CH}_{\bullet-2}(-) \otimes_{\mathbb{Z}} \mathbb{Z}_{(2)} \rightarrow \mathrm{CH}_{\bullet-2}(-) \otimes_{\mathbb{Z}} \mathbb{Q}\right)\right) \otimes_{\mathbb{Z}} \mathbb{Z} / 2,
$$

and when $S$ is of finite dimension,

$$
T_{3}: \mathrm{CH} \bullet(-) \otimes_{\mathbb{Z}} \mathbb{Z} / 2 \rightarrow\left(\operatorname{im}\left(\mathrm{CH}_{\bullet-3}(-) \otimes_{\mathbb{Z}} \mathbb{Z}_{(2)} \rightarrow \mathrm{CH}_{\bullet-3}(-) \otimes_{\mathbb{Z}} \mathbb{Q}\right)\right) \otimes_{\mathbb{Z}} \mathbb{Z} / 2 .
$$




\section{DETECTION BY REGULAR SCHEMES IN DEGREE TWO}

The operation $T_{2}$ is the second Steenrod square (modulo two-torsion), see [Hau12a, Proposition 8.1]. By contrast, the operation $T_{3}$ is not the usual third Steenrod square; nonetheless it can be used (together with $T_{2}$ and the first Steenrod square $T_{1}$ ) to define the third reduced Steenrod square, see [Hau12a, Remark 8.6].

Proceeding as in [Hau13, Section 6], but using operation $T_{2}$ instead of $T_{1}$, on can prove the following statement, which was already known in characteristic not two (see [Kar03]).

THEOREM 4.8. Let $\varphi$ be an anisotropic non-degenerate quadratic form over an arbitrary field. Let $\mathfrak{i}_{1}$ be the first Witt index of $\varphi$. If $\operatorname{dim} \varphi-\mathfrak{i}_{1}$ is not divisible by 4 , then $\mathfrak{i}_{1}$ is equal to 1 or 2 .

\section{Adem relation for the first Steenrod square}

In $[$ Hau13b, $\S 7]$ we gave a construction of the first Steenrod square, an operation

$$
\mathrm{Sq}_{1}: \mathrm{CH}_{\bullet}(X) \otimes_{\mathbb{Z}} \mathbb{Z} / 2 \rightarrow \mathrm{CH}_{\bullet-1}(X) \otimes_{\mathbb{Z}} \mathbb{Z} / 2,
$$

for $X$ quasi-projective over a field. This operation is a homological analog of $\mathrm{Sq}^{2}$ in topology; the Bockstein operation in motivic cohomology vanishes on Chow groups. We were not able to prove the relation $\mathrm{Sq}_{1} \circ \mathrm{Sq}_{1}=0$, and instead proved weaker relations. In this section, we use Theorem 2.1 to prove the relation $\mathrm{Sq}_{1} \circ \mathrm{Sq}_{1}=0$.

We first extend the definition of $\mathrm{Sq}_{1}$ to arbitrary schemes $X$ of finite type over a field. The next definition is close to that of a site, but will be more convenient for us, since our covers will always be singletons.

DeFinition 5.1. Let $\mathcal{C}$ be a category with fibre products. We say that $\mathcal{C}$ is a category with covers if are given a class of morphisms in $\mathcal{C}$, called covers, satisfying the following conditions:

- If $Y \rightarrow X$ is a cover and $X^{\prime} \rightarrow X$ is in $\mathcal{C}$, then $X^{\prime} \times_{X} Y \rightarrow X^{\prime}$ is a cover.

- If $Z \rightarrow Y$ and $Y \rightarrow X$ are covers, so is the composition $Z \rightarrow X$.

For a category $\mathcal{D}$, we denote by $\operatorname{Hom}(\mathcal{D}, A b)$ the category of functors $\mathcal{D} \rightarrow A$ b. Let $\mathcal{C}$ be a category with covers. The category $\operatorname{coShv}(\mathcal{C})$ is the full subcategory of $\operatorname{Hom}(\mathcal{C}, \mathrm{Ab})$ consisting of those $F: \mathcal{C} \rightarrow \mathrm{Ab}$, called abelian cosheaves on $\mathcal{C}$, such that for any cover $\pi: Y \rightarrow X$, the sequence of abelian groups

$$
F\left(Y \times_{X} Y\right) \stackrel{F\left(\pi_{1}\right)-F\left(\pi_{2}\right)}{\longrightarrow} F(Y) \stackrel{F(\pi)}{\longrightarrow} F(X) \rightarrow 0
$$

where $\pi_{1}, \pi_{2}: Y \times_{X} Y \rightarrow Y$ are the two projections, is exact.

Proposition 5.2. Let $\mathcal{C}$ be a category with covers and let $\mathcal{D}$ be a full subcategory. Assume that for every object $X \in \mathcal{C}$, there is a cover $Y \rightarrow X$ with $Y \in \mathcal{D}$. Then the natural functor $r: \operatorname{coShv}(\mathcal{C}) \rightarrow \operatorname{Hom}(\mathcal{D}, \mathrm{Ab})$ is fully faithful.

Proof. Let $\varphi: F \rightarrow G$ be a morphism in $\operatorname{coShv}(\mathcal{C})$ such that $r(\varphi)=0$. Let $X \in \mathcal{C}$. Choose a cover $\pi: Y \rightarrow X$ with $Y \in \mathcal{D}$. We have a commutative diagram

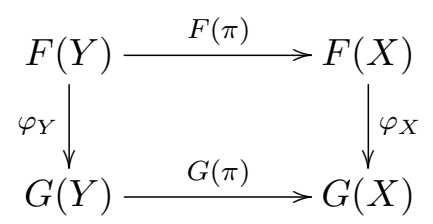




\section{OLIVIER HAUtion}

and $\varphi_{Y}=r(\varphi)_{Y}=0$, so that $\varphi_{X} \circ F(\pi)=0$. Since $F(\pi)$ is an epimorphism, it follows that $\varphi_{X}=0$. This proves that $\varphi=0$, hence $r$ is faithful.

Now, let $F, G \in \operatorname{coShv}(\mathcal{C})$ and let $\varphi: r(F) \rightarrow r(G)$ be a morphism of functors $\mathcal{D} \rightarrow$ Ab. Let $X \in \mathcal{C}$. Choose a cover $\pi: Y \rightarrow X$ with $Y \in \mathcal{D}$, and a cover $Z \rightarrow Y \times_{X} Y$ with $Z \in \mathcal{D}$. The two morphisms $p_{1}, p_{2}: Z \rightarrow Y \times_{X} Y \rightarrow Y$ in $\mathcal{C}$ belong to $\mathcal{D}$, since $\mathcal{D}$ is a full subcategory of $\mathcal{C}$. Therefore we have the commutative diagram with solid arrows

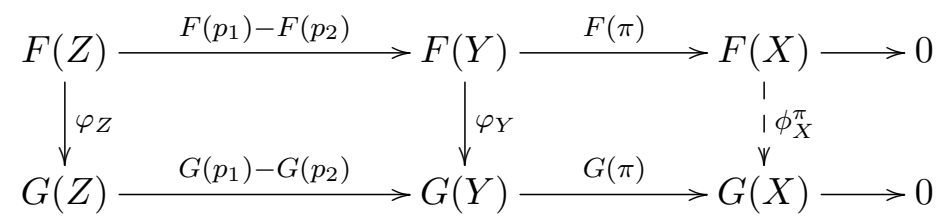

Since $F$ is an abelian cosheaf, the morphism $F(Z) \rightarrow F\left(Y \times_{X} Y\right)$ is surjective, and moreover the upper row is exact. So is the lower row, by the same argument. Thus there is a unique morphism $\phi_{X}^{\pi}$ fitting in the diagram.

For $i \in\{1,2\}$, let $\pi_{i}: Y_{i} \rightarrow X$ be a cover with $Y_{i} \in \mathcal{D}$. Choose a cover $Y^{\prime} \rightarrow Y_{1} \times_{X} Y_{2}$ with $Y^{\prime} \in \mathcal{D}$. For $i \in\{1,2\}$, the induced morphism $q_{i}: Y^{\prime} \rightarrow Y_{i}$ is in $\mathcal{D}$, and $\pi_{i} \circ q_{i}$ is a cover; moreover, we have two commutative diagrams
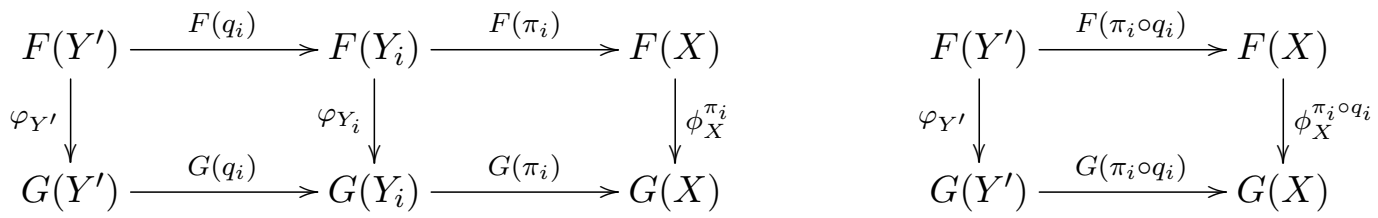

By the unicity of $\phi_{X}^{\pi}$, we see that $\phi_{X}^{\pi_{i}}=\phi_{X}^{\pi_{i} \circ q_{i}}$ for $i \in\{1,2\}$. Since the morphism $\pi_{i} \circ q_{i}$ is independent of $i$, so is the morphism $\phi_{X}^{\pi_{i}}$. This gives a well-defined morphism $\phi_{X}=\phi_{X}^{\pi}: F(X) \rightarrow$ $G(X)$ for every $X \in \mathcal{C}$. When $X \in \mathcal{D}$, we can take $\pi=\mathrm{id}_{X}$, so that $\phi_{X}=\varphi_{X}$.

Now, let $f: X^{\prime} \rightarrow X$ be a morphism in $\mathcal{C}$. Let $\pi: Y \rightarrow X$ be a cover with $Y \in \mathcal{D}$, and let $Y^{\prime} \rightarrow Y \times_{X} X^{\prime}$ be a cover with $Y^{\prime} \in \mathcal{D}$. The morphism $\pi^{\prime}: Y^{\prime} \rightarrow X^{\prime}$ is a cover, and $g: Y^{\prime} \rightarrow Y$ is in $\mathcal{D}$. Then

$$
\begin{aligned}
G(f) \circ \phi_{X^{\prime}} \circ F\left(\pi^{\prime}\right) & =G(f) \circ G\left(\pi^{\prime}\right) \circ \varphi_{Y^{\prime}} \\
& =G(\pi) \circ G(g) \circ \varphi_{Y^{\prime}} \\
& =G(\pi) \circ \varphi_{Y} \circ F(g) \\
& =\phi_{X} \circ F(\pi) \circ F(g) \\
& =\phi_{X} \circ F(f) \circ F\left(\pi^{\prime}\right) .
\end{aligned}
$$

Since $F\left(\pi^{\prime}\right)$ is an epimorphism, we get that $G(f) \circ \phi_{X^{\prime}}=\phi_{X} \circ F(f)$. Thus $\phi$ defines a morphism $F \rightarrow G$ in $\operatorname{coShv}(\mathcal{C})$, such that $r(\phi)=\varphi$. Therefore $r$ is full, concluding the proof.

Proposition 5.3. Let $C$ be an abelian group, $i$ an integer, and $S$ the spectrum of a field. The envelopes (see 1.6) define a class of covers on the category $\mathrm{Sch}_{S}$ for which the functor $\mathrm{CH}_{i}(-) \otimes_{\mathbb{Z}} C$ is an abelian cosheaf.

Proof. The first statement follows from [Ful98, Lemma 18.3 (1), (2)]. When $C=\mathbb{Z}$ the second statement is [Kim92, Theorem 1.8] (this does not use resolution of singularities). In general, it follows from the right-exactness of $-\otimes_{\mathbb{Z}} C$.

Proposition 5.4. Let $S$ be the spectrum of a field. The operation

$$
\mathrm{Sq}_{1}: \mathrm{CH}_{\bullet}(X) \otimes_{\mathbb{Z}} \mathbb{Z} / 2 \rightarrow \mathrm{CH}_{\bullet-1}(X) \otimes_{\mathbb{Z}} \mathbb{Z} / 2
$$




\section{DETECTION BY REGULAR SCHEMES IN DEGREE TWO}

defined in [Hau13b, $\S 7$ ] for quasi-projective $S$-schemes $X$ can be extended in a unique way compatible with proper push-forwards to all schemes $X$ of finite type over $S$. Moreover, the resulting operation, which we denote again by $\mathrm{Sq}_{1}$, is compatible with restrictions to open subschemes.

Proof. By Proposition 5.3 with $C=\mathbb{Z} / 2$ and the existence of Chow's envelopes (see 1.6), the conditions of Proposition 5.2 are satisfied for $\mathcal{C}=\mathrm{Sch}_{S}$ with covers given by envelopes and $\mathcal{D}$ the full subcategory of quasi-projective $S$-schemes. Therefore the operation $\mathrm{Sq}_{1}$ of [Hau13b, $\S 7$ ] extends uniquely to a morphism $\mathrm{Sq}_{1}$ of functors $\mathrm{Sch}_{S} \rightarrow$ Ab.

Let $u: U \rightarrow X$ be an open immersion. Let $\pi: Y \rightarrow X$ be a Chow envelope over $S$, and form the cartesian square

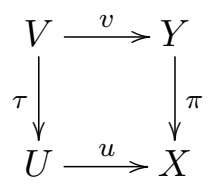

Then $v$ is an open immersion between quasi-projective $S$-schemes, hence $\mathrm{Sq}_{1} \circ v^{*}=v^{*} \circ \mathrm{Sq}_{1}$ by [Hau13b, Proposition 7.6]. We have

$$
\mathrm{Sq}_{1} \circ u^{*} \circ \pi_{*}=\mathrm{Sq}_{1} \circ \tau_{*} \circ v^{*}=\tau_{*} \circ \mathrm{Sq}_{1} \circ v^{*}=\tau_{*} \circ v^{*} \circ \mathrm{Sq}_{1}=u^{*} \circ \pi_{*} \circ \mathrm{Sq}_{1}=u^{*} \circ \mathrm{Sq}_{1} \circ \pi_{*} .
$$

Since $\pi_{*}$ is an epimorphism, it follows that $\mathrm{Sq}_{1} \circ u^{*}=u^{*} \circ \mathrm{Sq}_{1}$, hence $\mathrm{Sq}_{1}$ is compatible with restrictions to open subschemes.

Lemma 5.5. Let $X$ be a regular scheme, quasi-projective over a field. Then

$$
\mathrm{Sq}_{1} \circ \mathrm{Sq}_{1}[X]=0 \in \mathrm{CH}(X) \otimes_{\mathbb{Z}} \mathbb{Z} / 2 .
$$

Proof. Let $T_{X} \in K_{0}(X)$ be the virtual tangent bundle of $X$ and let $L_{X}$ be a line bundle over $X$ representing the image of $T_{X}$ under the group homomorphism det: $K_{0}(X) \rightarrow \operatorname{Pic}(X)$ (the sheaf of sections of $L_{X}$ is the canonical sheaf of $\left.X\right)$. Let $s: X \hookrightarrow L_{X}$ be its zero section. Then the first Chern class operators satisfy

$$
c_{1}\left(T_{X}\right)=c_{1}\left(L_{X}\right)=s^{*} \circ s_{*}: \mathrm{CH}(X) \rightarrow \mathrm{CH}(X) .
$$

By [Hau13b, (11)], we have $\operatorname{Sq}_{1}[X]=c_{1}\left(T_{X}\right)[X]$ in $\mathrm{CH}(X) \otimes_{\mathbb{Z}} \mathbb{Z} / 2$. Using [Hau13b, Proposition 7.6], we have in $\mathrm{CH}(X) \otimes_{\mathbb{Z}} \mathbb{Z} / 2$

$$
\begin{aligned}
\mathrm{Sq}_{1} \circ \mathrm{Sq}_{1}[X] & =\mathrm{Sq}_{1} \circ s^{*} \circ s_{*}[X] \\
& =s^{*} \circ \mathrm{Sq}_{1} \circ s_{*}[X]+c_{1}\left(L_{X}\right) \circ s^{*} \circ s_{*}[X] \\
& =s^{*} \circ s_{*} \circ \mathrm{Sq}_{1}[X]+c_{1}\left(L_{X}\right) \circ s^{*} \circ s_{*}[X] \\
& =s^{*} \circ s_{*} \circ c_{1}\left(L_{X}\right)[X]+c_{1}\left(L_{X}\right) \circ s^{*} \circ s_{*}[X] \\
& =c_{1}\left(L_{X}\right) \circ c_{1}\left(L_{X}\right)[X]+c_{1}\left(L_{X}\right) \circ c_{1}\left(L_{X}\right)[X] \\
& =0 \bmod 2 .
\end{aligned}
$$

Proposition 5.6. We have $\mathrm{Sq}_{1} \circ \mathrm{Sq}_{1}=0$.

Proof. We apply Lemma 3.1 with $S=T$ the spectrum of a field, and with $G$, respectively $H$, given by Example 3.3, with $C=\mathbb{Z} / 2$ and $j=d$, respectively $j=d-2$, for the operation

$$
\rho_{X}=\mathrm{Sq}_{1} \circ \mathrm{Sq}_{1}: \mathrm{CH}_{d}(X) \otimes_{\mathbb{Z}} \mathbb{Z} / 2 \rightarrow \mathrm{CH}_{d-2}(X) \otimes_{\mathbb{Z}} \mathbb{Z} / 2 .
$$

Then conditions (iii) and (iv) follow from Proposition 5.4, and (v) from Lemma 5.5. 


\section{Olivier Haution}

\section{Bivariant Chow group}

In this section we consider the bivariant Chow group $\mathrm{A}(X \rightarrow T)$. Of special interest is the group $\mathrm{A}\left(\mathrm{id}_{X}\right)$, which can be considered as the universal cohomology theory acting on Chow groups (it has a ring structure and is contravariant for arbitrary morphisms of finite type). More generally, when $X$ is a closed subscheme of $T$, the non-unital ring $\mathrm{A}(X \rightarrow T)$ corresponds to cohomology of $T$ with support in $X$. We are interested in the commutativity of this ring, and in Poincaré duality-type statements.

We first define bivariant Chow groups following [Ful98, $\S 17, \S 20]$, with the difference that we require bivariant classes to be compatible with smooth pull-backs, as opposed to arbitrary flat pull-backs (see, however, Remark 6.9 (ii) and Theorem 6.11 (iii)).

Let $S$ be a regular scheme and let $X \rightarrow T \rightarrow S$ be morphisms of finite type. For $m \in \mathbb{Z}$, we consider the set $\mathrm{B}^{m}(X \rightarrow T)$ of morphisms $\alpha$ of functors $\operatorname{Sch}_{T} \rightarrow \mathrm{Ab}$

$$
\mathrm{CH}_{\bullet}(-) \rightarrow \mathrm{CH}_{\bullet}-m\left(-\times_{T} X\right), \quad x \mapsto \alpha \cap x .
$$

Let $Y$ be a scheme of finite type over $T$. Any element $\alpha \in \mathrm{B}^{m}(X \rightarrow T)$ induces an element of $\mathrm{B}^{m}\left(X \times_{T} Y \rightarrow Y\right)$ which we still denote by $\alpha$. When $g: X \rightarrow T$ is a flat morphism of constant relative dimension $-r$, or a regular closed embedding of codimension $r$, there is an orientation class $[g] \in \mathrm{B}^{r}(X \rightarrow T)$ corresponding to the fact that base changes of such morphisms admit functorial (refined) pull-backs (see [Ful98, § 17.4]). There is a product

$$
\mathrm{B}^{m}(X \rightarrow T) \otimes_{\mathbb{Z}} \mathrm{B}^{n}(Y \rightarrow T) \rightarrow \mathrm{B}^{m+n}\left(X \times_{T} Y \rightarrow T\right),
$$

written $a \otimes b \mapsto a \cdot b$.

The group $\mathrm{A}^{m}(X \rightarrow T)$ consists of the elements $\alpha \in \mathrm{B}^{m}(X \rightarrow T)$, called bivariant classes, such that $[f] \cdot \alpha=\alpha \cdot[f]$ for $f$ any smooth morphism or regular closed embedding. We denote by $\mathrm{A}(X \rightarrow T)$ the direct sum of the groups $\mathrm{A}^{m}(X \rightarrow T)$ over the integers $m \in \mathbb{Z}$. If $X \rightarrow T$ has relative dimension at most $r$ (Example 3.4), then $\mathrm{B}^{m}(X \rightarrow T)=\mathrm{A}^{m}(X \rightarrow T)=0$ for $m<-r$.

The product of bivariant classes is a bivariant class, and so is $[g]$ for $g$ as above (flat or regular closed embedding). When $h: Y \rightarrow X$ is a morphism in $\operatorname{Sch}_{T}$, there is a morphism $h_{*}: \mathrm{A}^{m}(Y \rightarrow T) \rightarrow \mathrm{A}^{m}(X \rightarrow T)$.

REMARK 6.1. An element $\alpha \in \mathrm{B}^{m}(X \rightarrow T)$ such that $[f] \cdot \alpha=\alpha \cdot[f]$, for $f$ any smooth morphism or effective Cartier divisor with trivial normal bundle, is a bivariant class. This can be seen using deformation to the normal cone (see the proof of [Ful98, Theorem 17.1]).

EXAmple 6.2. Let $X \rightarrow T$ be a closed embedding and let $\mathcal{E}_{\bullet}$ be a bounded complex of locally free coherent $\mathcal{O}_{T}$-modules which is exact off $X$. The $n$th localised Chern class $c_{n}\left(\mathcal{E}_{\bullet}\right)$ is an element of $\mathrm{A}^{n}(X \rightarrow T)$, see [Ful98, Example 18.1.3, $\left.\S 20\right]$.

ExAmple 6.3. Let $X \rightarrow T$ be a closed embedding. Let $K_{n}\left(\mathcal{O}_{T}\right)$ be the Zariski sheaf on $T$ associated with the $n$th Quillen $K$-group of vector bundles. We describe a morphism

$$
H_{X}^{n}\left(T, K_{n}\left(\mathcal{O}_{T}\right)\right) \rightarrow \mathrm{A}^{n}(X \rightarrow T) .
$$

For any scheme $Y$ of finite type over $T$, there is a morphism (see [Gil81, $\S 7, \S 8]$ )

$$
H_{X}^{n}\left(T, K_{n}\left(\mathcal{O}_{T}\right)\right) \otimes \mathrm{CH}_{d}(Y) \rightarrow \mathrm{CH}_{d-n}\left(X \times_{T} Y\right) .
$$

The projection formula [Gil81, Theorem 8.8] says that it is compatible with proper push-forwards; therefore any element $\alpha \in H_{X}^{n}\left(T, K_{n}\left(\mathcal{O}_{T}\right)\right)$ defines an element of $\mathrm{B}^{n}(X \rightarrow T)$, which we also 


\section{DETECTION BY REGULAR SCHEMES IN DEGREE TWO}

denote by $\alpha$. When $f$ is smooth (or more generally flat of constant relative dimension), the formula $[f] \cdot \alpha=\alpha \cdot[f]$ is easily verified.

To show that $\alpha$ defines an element of $\mathrm{A}^{n}(X \rightarrow T)$, it will suffice by Remark 6.1 to consider an effective Cartier divisor $f: D \rightarrow T$ with trivial normal bundle, and prove that $\alpha \cdot[f]=[f] \cdot \alpha$. The invertible sheaf $\mathcal{O}_{T}(D)$, being trivial off $D$, gives rise to an element $\delta \in H_{D}^{1}\left(T, \mathcal{O}_{T}^{\times}\right)=$ $H_{D}^{1}\left(T, K_{1}\left(\mathcal{O}_{T}\right)\right)$.

We claim that $[f]=\delta$. Indeed, it is enough to check that $f^{!}[Z]=\delta \cap[Z] \in \mathrm{CH}(Z)$ when $Z$ is an integral scheme of finite type over $T$. Assume first that $Z \rightarrow T$ factors through $f$. Since $f$ has trivial normal bundle, $\delta$ restricts to zero in $H_{D}^{1}\left(D, K_{1}\left(\mathcal{O}_{D}\right)\right)$, hence we have $\delta \cap[Z]=0$. On the other hand, $f^{!}[Z]=0$ by, for example, [Ful98, Proposition 2.3 (e)]. Otherwise $f$ induces an effective Cartier divisor with trivial normal bundle on $Z$, and we are reduced to assuming $T=Z$. Then $f^{!}[T]=[D]$ and $\delta \cap[T]=[D]$ (this computation is carried out in [Gil87, $\left.\S 2\right]$ ).

The map

$$
\oplus_{n, Z} H_{Z}^{n}\left(T, K_{n}\left(\mathcal{O}_{T}\right)\right) \rightarrow \oplus_{n, Z} \mathrm{~B}^{n}(Z \rightarrow T),
$$

where $n \in \mathbb{N}$ and $Z$ runs over the closed subschemes of $T$, is a ring morphism by [Gil81, Theorem 8.2]. It follows from the anti-commutativity of Quillen $K$-theory that the ring on the left is commutative. Thus the elements of the image of this morphism commute with one another, and in particular $\alpha$ commutes with $\delta=[f]$, as required.

Lemma 6.4. Let $S$ be a regular excellent scheme, and $X$ an integral closed subscheme of $S$. Assume $n=-\operatorname{dim}_{S} X>0$. There is a finite resolution $\mathcal{E}_{\bullet}$ of $\mathcal{O}_{X}$ by locally free coherent $\mathcal{O}_{S^{-}}$ modules, and for any such complex $\mathcal{E}_{\bullet}$, we have $c_{n}\left(\mathcal{E}_{\bullet}\right) \cap[S]=(-1)^{n-1}(n-1) ! \cdot[X]$ in $\mathrm{CH}_{-n}(X)$.

Proof. The resolution $\mathcal{E}_{\bullet}$ exists because $S$ is regular, and, moreover, the class $c_{n}\left(\mathcal{E}_{\bullet}\right)$ is independent of the choice of that resolution. The singular locus $\operatorname{Sing} X$ is closed and not dense in $X$, because $X$ is quasi-excellent and integral. It follows from the localisation sequence that the morphism $\mathrm{CH}_{-n}(X) \rightarrow \mathrm{CH}_{-n}(X-\operatorname{Sing} X)$ is an isomorphism. We may therefore replace $S$ and $X$ by the respective complements of $\operatorname{Sing} X$, and assume that $X$ is regular. Then $X \rightarrow S$ is a regular closed embedding [EGA IV, (19.1.1)]; we let $N$ be its normal bundle. We consider the deformation to the normal cone construction [Ful98, §6.1]

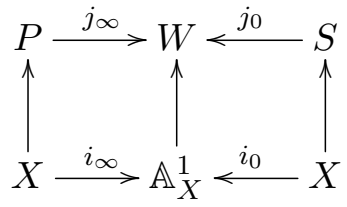

where $P=\mathbb{P}(N \oplus 1)$ and $W$ is the blow-up of $\mathbb{A}_{S}^{1}$ along $X$ via the composition $X \stackrel{i_{0}}{\rightarrow} \mathbb{A}_{X}^{1} \rightarrow \mathbb{A}_{S}^{1}$. Each of the schemes $P$ and $\mathbb{A}_{X}^{1}$, being smooth over $X$, is regular by [EGA IV, (17.5.8, (iii))]. The scheme $W$ is regular ( $W-P$ is regular, being an open subscheme of $X$, and $W$ is regular at every point of $P$ by [EGA IV, (19.1.1)]), hence we can find a finite resolution $E_{\bullet}$ of $\mathcal{O}_{\mathbb{A}_{X}^{1}}$ by locally free coherent $\mathcal{O}_{W}$-modules. Then $j_{\infty}^{*} E_{\bullet}$ and $j_{0}^{*} E_{\bullet}$ are finite resolutions of $\mathcal{O}_{X}$ by locally free coherent $\mathcal{O}_{P}$-modules and $\mathcal{O}_{S}$-modules, respectively. Using the transversality of the squares in the diagram above and the relation $i_{0}^{*}=i_{\infty}^{*}: \mathrm{CH}_{-n}\left(\mathbb{A}_{X}^{1}\right) \rightarrow \mathrm{CH}_{-n}(X)$, we have in $\mathrm{CH}_{-n}(X)$

$$
c_{n}\left(j_{0}^{*} E_{\bullet}\right) \cap[S]=i_{0}^{*}\left(c_{n}\left(E_{\bullet}\right) \cap[W]\right)=i_{\infty}^{*}\left(c_{n}\left(E_{\bullet}\right) \cap[W]\right)=c_{n}\left(j_{\infty}^{*} E_{\bullet}\right) \cap[P] .
$$

Thus we may replace $S$ by $P$ and assume that $f: X \rightarrow S$ has a section $p: S \rightarrow X$. Then we have 


\section{OLIVIER HAUtion}

in $\mathrm{CH}_{-n}(S)$ (see the proof of [Ful98, Proposition 18.1 (a)])

$$
f_{*}\left(c_{n}\left(\mathcal{E}_{\bullet}\right) \cap[S]\right)=\sum_{i}(-1)^{i} c_{n}\left(\mathcal{E}_{i}\right)[S]=c_{n}\left(f_{*}\left[\mathcal{O}_{X}\right]\right)[S] .
$$

This cycle coincides with $(-1)^{n-1}(n-1) ! \cdot[X]$ by [Ful98, Example 15.3.1, $\left.\S 20\right]$, and we conclude by applying $p_{*}: \mathrm{CH}_{-n}(S) \rightarrow \mathrm{CH}_{-n}(X)$.

TheOREM 6.5. Let $S$ be a regular excellent scheme and let $X$ be a closed subscheme of $S$. Then for $n \leqslant 2$, the association $\alpha \mapsto \alpha \cap[S]$ induces an isomorphism

$$
\mathrm{A}^{n}(X \rightarrow S) \rightarrow \mathrm{CH}_{-n}(X) .
$$

Proof. Let $\alpha \in \mathrm{A}^{n}(X \rightarrow S)$ be such that $\alpha \cap[S]=0$. Let $r: R \rightarrow S$ be a quasi-projective morphism, with $R$ regular. By Lemma 4.4, we can write $r=p \circ i$, with $i$ a regular closed embedding and $p$ a smooth morphism. We have in $\mathrm{CH}\left(R \times{ }_{S} X\right)$

$$
\alpha \cap[R]=(\alpha \cdot[i] \cdot[p]) \cap[S]=([i] \cdot[p] \cdot \alpha) \cap[S]=0 .
$$

Let $d$ be an integer. For any scheme $Y$ of finite type over $S$, we let $\rho_{Y}: \mathrm{CH}_{d}(Y) \rightarrow \mathrm{CH}_{d-n}\left(Y \times_{S} X\right)$ be defined by $\rho_{Y}(x)=\alpha \cap x$. Then $\rho$ satisfies the conditions of Lemma 3.1 for $T=S$, with $G$ as in Example $3.3(C=\mathbb{Z}, j=d)$ and $H$ as in Example $3.4(r=0, j=d-n)$. This proves that $\alpha=0$, showing injectivity.

Let $i: Z \rightarrow X$ be a closed embedding, with $Z$ integral and $\operatorname{dim}_{S} Z=-n \leqslant 0$. There is a bounded complex $\mathcal{E}_{\bullet}$ of locally free coherent $\mathcal{O}_{S}$-modules, exact off $Z$, such that $c_{n}\left(\mathcal{E}_{\bullet}\right) \cap[S]=$ $(-1)^{n-1} \cdot[Z] \in \mathrm{CH}_{-n}(Z)$; this follows from Lemma 6.4 when $n \in\{1,2\}$, and we may take $\mathcal{E}_{\bullet}=\mathcal{O}_{Z}[-1]$ when $n=0$. Therefore $\alpha=i_{*}\left(c_{n}\left(\mathcal{E}_{\bullet}\right)\right)$ is an antecedent of the class $(-1)^{n-1} \cdot[Z]$ under the map of the statement. Since such classes generate the group $\mathrm{CH}_{-n}(X)$, we have proved surjectivity.

Let us record the following statement, obtained in the course of the proof above.

Lemma 6.6. Let $S$ be a regular excellent scheme and let $X$ be a closed subscheme of $S$. For $n \leqslant 2$, the group $\mathrm{A}^{n}(X \rightarrow S)$ is generated by the elements $i_{*}\left(c_{n}\left(\mathcal{E}_{\bullet}\right)\right)$, where $i: Z \rightarrow X$ is a closed embedding, and $\mathcal{E}_{\bullet}$ a bounded complex of locally free coherent $\mathcal{O}_{S}$-modules, which is exact off $Z$.

Proposition 6.7. Let $S$ be a regular excellent scheme, let $X$ be a closed subscheme of $S$, and let $Y$ be a scheme of finite type over $S$. Then for any $\alpha \in \mathrm{A}^{n}(X \rightarrow S)$ with $n \leqslant 2$ and $\beta \in \mathrm{A}(Y \rightarrow S)$, we have $\alpha \cdot \beta=\beta \cdot \alpha$.

Proof. We may assume $\alpha=i_{*}\left(c_{n}\left(\mathcal{E}_{\bullet}\right)\right)$, as in Lemma 6.6. Since the construction of the class $c_{n}\left(\mathcal{E}_{\bullet}\right)$ uses only proper push-forwards, smooth pull-backs, and Gysin maps, one checks that $\beta \cdot c_{n}\left(\mathcal{E}_{\bullet}\right)=c_{n}\left(\mathcal{E}_{\bullet}\right) \cdot \beta$. But $\beta$ is compatible with $i_{*}$, by definition of $\mathrm{B}^{m}(Y \rightarrow S)$.

Proposition 6.8. Let $S$ be a regular excellent scheme and let $Y \rightarrow T \rightarrow S$ be morphisms of finite type. Let $X$ be a closed subscheme of $T$. Assume that $X \times_{T} Y \rightarrow T$ has relative dimension at most $r$ (Example 3.4). For any $\alpha \in \mathrm{A}^{n}(X \rightarrow T)$ and $\beta \in \mathrm{A}^{m}(Y \rightarrow T)$, with $m+n+r \leqslant 2$ and $n \leqslant 2$, we have $\alpha \cdot \beta=\beta \cdot \alpha$.

Proof. Let $d$ be an integer. For any scheme $B$ of finite type over $T$, we let $\rho_{B}: \mathrm{CH}_{d}(B) \rightarrow$ $\mathrm{CH}_{d-m-n}\left(B \times_{T} X \times_{T} Y\right)$ be defined by $\rho_{B}(x)=(\alpha \cdot \beta-\beta \cdot \alpha) \cap x$. Let $R$ be a regular scheme of finite type over $T$. We have $\rho_{R}[R]=0$ by Proposition 6.7 since $n \leqslant 2$. We conclude using Lemma 3.1, with $G$ as in Example $3.3(C=\mathbb{Z}, j=d)$ and $H$ as in Example $3.4(j=d-r-m-n)$. 


\section{DETECTION BY REGULAR SCHEMES IN DEGREE TWO}

REMARK 6.9. Proposition 6.8 applies in particular in the following situations.

(i) The scheme $Y$ is a closed subscheme of $T$ and $m=n=1, r=0$. This says that elements of degree one commute in the ring $\bigoplus_{Z} \mathrm{~A}(Z \rightarrow T)$, where $Z$ runs over the closed subschemes of $T$.

(ii) The morphism $f: Y \rightarrow T$ is flat of constant relative dimension $r=-m, \beta=[f]$, and $n \leqslant 2$. This says that any element of $\mathrm{A}^{n}(X \rightarrow T)$ with $n \leqslant 2$ is automatically compatible with flat pull-backs. Therefore our definition of $\mathrm{A}^{n}(X \rightarrow T)$ agrees with that of [Ful98, $\left.\S 17\right]$ when $n \leqslant 2$ and $X \rightarrow T$ is a closed embedding.

REMARK 6.10. When $S$ is a field of characteristic zero, bivariant classes of arbitrary degrees commute [Ful98, Example 17.4.4].

When $S$ is smooth over a field and $X$ is a scheme of finite type over $S$, the map $\mathrm{A}(X \rightarrow S) \rightarrow$ $\mathrm{CH}(X)$ is known to be an isomorphism [Ful98, Propositions 17.3.1 and 17.4.2].

The results of this section can be improved in characteristic zero, as follows.

TheOREm 6.11. Let $S$ be a regular excellent $\mathbb{Q}$-scheme and let $X$ be a scheme of finite type over $S$.

(i) The morphism $\mathrm{A}(X \rightarrow S) \rightarrow \mathrm{CH}(X)$ is an isomorphism.

(ii) If $A$ and $B$ are schemes of finite type over $X$, and $\alpha \in \mathrm{A}(A \rightarrow X), \beta \in \mathrm{A}(B \rightarrow X)$, then we have $\alpha \cdot \beta=\beta \cdot \alpha$.

(iii) For any scheme $Y$ finite type over $X$, the group $\mathrm{A}(Y \rightarrow X)$ coincides with that of [Ful98, $\S 17, \S 20]$.

Proof. We obtain the injectivity in assertion (i) by proceeding as in the proof of Theorem 6.5 , using Remark 3.2.

To prove the surjectivity in assertion (i), it will be sufficient to assume that $X$ is integral, and find an element $\alpha \in \mathrm{A}(X \rightarrow S)$ such that $\alpha \cap[S]=[X]$. By Chow's lemma [EGA II, (5.6.1)], we may assume that $X$ is quasi-projective over $S$, so that by Lemma 4.4, the morphism $X \rightarrow S$ decomposes as $p \circ i$, where $i: X \rightarrow W$ is a closed embedding and $p: W \rightarrow S$ is smooth. Then for any $\beta \in \mathrm{A}(X \rightarrow W)$, we have $\beta \cap[W]=(\beta \cdot[p]) \cap[S]$, so that we may replace $S$ by $W$ (the latter is regular by [EGA IV, (17.5.8, (iii))]), and assume that $X \rightarrow S$ is a closed embedding. Since Gersten's conjecture is true for the local rings of $S$ by [Pan03], the composition

$$
H_{X}^{n}\left(S, K_{n}\left(\mathcal{O}_{S}\right)\right) \rightarrow \mathrm{A}^{n}(X \rightarrow S) \rightarrow \mathrm{CH}_{-n}(X)
$$

is an isomorphism (this is Bloch's formula, see for instance [Gil81, $\S 8$ and Corollary 7.20]). This proves the surjectivity in assertion (i).

Let us mention that we actually proved that any element of $\mathrm{A}^{n}(X \rightarrow S)$ decomposes as $\pi_{*}(\alpha \cdot[p])$, where $\pi: X^{\prime} \rightarrow X$ is a proper morphism, $p: W \rightarrow S$ is smooth, $X^{\prime}$ is a closed subscheme of $W$, and $\alpha \in H_{X^{\prime}}^{n+\operatorname{dim}_{S} W}\left(W, K_{n+\operatorname{dim}_{S} W}\left(\mathcal{O}_{W}\right)\right)$.

If $A$ and $B$ are two closed subschemes of $S$, and $\alpha \in H_{A}^{n}\left(S, K_{n}\left(\mathcal{O}_{S}\right)\right), \beta \in H_{B}^{m}\left(S, K_{m}\left(\mathcal{O}_{S}\right)\right)$, we have $\alpha \cdot \beta=\beta \cdot \alpha$ in $\mathrm{A}^{m+n}(A \cap B \rightarrow S$ ) (see the last paragraph of Example 6.3). From the decomposition $\pi_{*}(\alpha \cdot[p])$ mentioned above, we obtain assertion (ii) under the additional assumption that $X$ is regular. Then we remove this assumption by reasoning as in the proof of Proposition 6.8 (using Remark 3.2).

Finally, assertion (iii) follows from assertion (ii), as in Remark 6.9 (ii). 


\section{Olivier Haution}

\section{ACKNOWLEDGEMENTS}

I am grateful to the referee for his remarks and explanations, which helped improve the exposition.

\section{REFERENCES}

Bro03 P. Brosnan, Steenrod operations in Chow theory, Trans. Amer. Math. Soc. 355 (2003), no. 5, 1869-1903 (electronic).

Ful98 W. Fulton, Intersection theory, volume 2 of Ergebnisse der Mathematik und ihrer Grenzgebiete. 3. Folge. A Series of Modern Surveys in Mathematics, Springer-Verlag, Berlin, second edition, 1998.

Gil81 H. Gillet Riemann-Roch theorems for higher algebraic K-theory, Adv. in Math. 40 (1981), no. 3, 203-289.

Gil87 H. Gillet. K-theory and intersection theory revisited, K-Theory 1 (1987), no. 4, 405-415.

EGA I A. Grothendieck, Éléments de géométrie algébrique I, Le langage des schémas, Publ. Math. Inst. Hautes Études Sci. 4 (1960).

EGA II A. Grothendieck, Éléments de géométrie algébrique II, Étude globale élémentaire de quelques classes de morphismes, Publ. Math. Inst. Hautes Études Sci. 8 (1961).

EGA IV A. Grothendieck, Éléments de géométrie algébrique IV, Étude locale des schémas et des morphismes de schémas, Publ. Math. Inst. Hautes Études Sci. 20 (1964), 24 (1965), 28 (1966) and 32 (1967).

Hau12a O. Haution, Integrality of the Chern character in small codimension, Adv. Math. 231 (2012), no. 2, 855-878. http://dx.doi.org/10.1016/j.aim.2012.04.030

Hau12b O. Haution, Reduced Steenrod operations and resolution of singularities, J. K-Theory 9 (2012), no. 2, 269-290. http://dx.doi.org/10.1017/is011006030jkt162

Hau13 O. Haution, On the first Steenrod square for Chow groups, Amer. J. Math. 135 (2013), no. 1, 53-63. http://dx.doi.org/10.1353/ajm.2013.0003

Hau13b O. Haution, Duality and the topological filtration, Math. Ann. 357 (2013), no. 4, 1425-1454. http://dx.doi.org/10.1007/s00208-013-0956-8

Hir64 H. Hironaka, Resolution of singularities of an algebraic variety over a field of characteristic zero. I, II, Ann. of Math. (2) 79 (1964), 109-203, ibid. (2) 79 (1964) 205-326.

ILO12 L. Illusie, Y. Laszlo, and F. Orgogozo, Travaux de Gabber sur l'uniformisation locale et la cohomologie étale des schémas quasi-excellents. Séminaire à l'École polytechnique 2006-2008, with the collaboration of F. Déglise, A. Moreau, V. Pilloni, M. Raynaud, J. Riou, B. Stroh, and M. Temkin, Astérisque 363-364, Soc. Math. de France, Paris, 2014.

Kar03 N. A. Karpenko, On the first Witt index of quadratic forms, Invent. Math. 153 (2003), no. 2, 455-462. http://dx.doi.org/10.1007/s00222-003-0294-7

Kim92 S. Kimura, Fractional intersection and bivariant theory, Comm. Algebra 20 (1992), no. 1, 285302.

Lip78 J. Lipman, Desingularization of two-dimensional schemes, Ann. Math. (2) 107 (1978), no. 1, 151-207.

Pan03 I. A. Panin, The equicharacteristic case of the Gersten conjecture, Tr. Mat. Inst. Steklova 241 (2003), Teor. Chisel, Algebra i Algebr. Geom., 169-178, translation in Proc. Steklov Inst. Math. 2003 241, no. 2, 154-163.

SGA 6 Séminaire de Géométrie algébrique du Bois-Marie, 1966-1967 (SGA 6), Théorie des intersections et théorème de Riemann-Roch. Lecture Notes in Mathematics, Vol. 225, Springer-Verlag, Berlin, 1971.

Sou85 C. Soulé. Opérations en K-théorie algébrique, Canad. J. Math. 37 (1985), no. 3, 488-550. 


\section{DETECTION BY REGULAR SCHEMES IN DEGREE TWO}

Tem08 M. Temkin, Desingularization of quasi-excellent schemes in characteristic zero, Adv. Math. 219 (2008), no. 2, 488-522. http://dx.doi.org/10.1016/j.aim.2008.05.006

Tem12 M. Temkin, Functorial desingularization of quasi-excellent schemes in characteristic zero: the nonembedded case, Duke Math. J. 161 (2012), no. 11, 2207-2254. http://dx.doi.org/10. 1215/00127094-1699539

Voe03 V. Voevodsky, Reduced power operations in motivic cohomology, Publ. Math. Inst. Hautes Études Sci. 98 (2003), 1-57. http://dx.doi.org/10.1007/s10240-003-0009-z

Olivier Haution olivier.haution@gmail.com

Mathematisches Institut, Ludwig-Maximilians-Universität München, Theresienstr. 39, D-80333

München, Germany 